\title{
TRATAMENTO DE RATOS, EXPERIMENTALMENTE INFECTADOS PELO STRONGYLOIDES VENEZUELENSIS, ATRAVÉS DA IVERMECTINA ADMINISTRADA POR VIA ORAL
}

\author{
Vicente Amato Neto, Fábio Luís Carignani, Luís Matsubara \\ e Lúcia Maria Almeida Braz
}

\begin{abstract}
Em modelo experimental, baseado na infecção de ratos pelo Strongyloides venezuelensis, foi avaliada a atividade terapêtica de duas preparações de ivermectina, para usos veterinário e humano. Houve interesse em verificar a efetividade em relação a vermes adultos e formas larvárias. A administração dos fármacos ocorreu sempre por via oral e a posologia correspondeu à dose única de $0,2 \mathrm{mg} / \mathrm{kg}$. Considerados os vermes adultos e as formas larvárias, o produto para emprego veterinário propiciou eliminações expressas pelas porcentagens de 98,0\% e 84,2\%; quanto à outra preparação, as taxas situaram-se em 59,3\% e 73,0\%, respectivamente. O estudo revelou, então, utilidade do anti-helmíntico quando usada a via oral e, também, mostrou significativa ação sobre as formas larvárias, certamente valiosa quando vigente a modalidade disseminada da estrongiloidiase.
\end{abstract}

Palavras-chaves: Strongyloides venezuelensis. Infecção experimental em ratos. Ivermectina. Tratamento por via oral.

A estrongiloidíase continua sendo expressivo problema entre as helmintíases, influindo para isso sobretudo a capacidade de autoinfecção por parte do agente causal, a ocasional resistência à erradicação terapêutica, a dificuldade para eliminar as formas larvárias e o risco de hiperinfecção em imunodeprimidos 7 .

Extremamente ativa em baixas dosagens contra uma ampla variedade de nematóides e artrópodes, a ivermectina, derivada da avermectina B1, que é lactona macrocíclica semi-sintética produzida pelo Streptomyces avermitilis 346 . Informes sugerem que ela causa paralisia em nematóides e artrópodes, interferindo na transmissão de sinais nervosos por aumento da permeabilidade da membrana celular para o íon cloreto, atuante no neurotransmissor ácido gama-amino-butírico (GABA)3 6. Convém salientar que esse medicamento tem sido usado para benefício da saúde animal em muitos países, desde 1985146.

Laboratório de Investigação Médica-Parasitologia do Hospital das Clínicas, Faculdade de Medicina da Universidade de São Paulo, São Paulo, SP, Brasil.

Endereço para correspondência: Prof. Vicente Amato Neto. Av. Dr. Enéas de Carvalho Aguiar 500, 05403-000 São Paulo, SP. Fax: (011) 881-8158.

Recebido para publicação em 31/10/96.
Estudos concernentes à ivermectina, usada parenteralmente, demonstraram eficácia antiparasitária, com atividade antilarvária no modelo experimental que utiliza ratos infectados pelo Strongyloides venezuelensis 5 , sendo que doses de até $200 \mu \mathrm{g} / \mathrm{kg}$ foram também bem toleradas e eficazes em seres humanos 156.

Com o intuito de contribuir para melhor conhecimento da ação terapêutica da ivermectina, consideramos adequado empreender avaliação experimental em ratos parasitados pelo Strongyloides venezuelensis, havendo emprego por via oral e apreciação do comportamento perante larvas e vermes adultos.

\section{MATERIAL E MÉTODOS}

Utilizamos ratos albinos, fêmeas, da linhagem Wistar, com peso corporal de 150 a $180 \mathrm{~g}$, fornecidos pelo Biotério Central, da Faculdade de Medicina da Universidade de São Paulo.

A cepa de $S$. venezuelensis foi obtida a partir de fezes de Rattus rattus novergicus, capturados pelo Departamento de Controle de Zoonoses e Vigilância Sanitária, da Prefeitura do Município de São Paulo. Isolamos a linhagem segundo metodologia indicada por Brener e Chaia ${ }^{2}$ e, para manutenção, efetuamos repiques sucessivos, no Laboratório de Investigação MédicaParasitologia do Hospital das Clínicas, da 
Amato Neto V, Carignani FL, Matsubara L, Braz LMA. Tratamento de ratos, experimentalmente infectados pelo Strongyloides venezuelensis, através da ivermectina administrada por via oral. Revista da Sociedade Brasileira de Medicina Tropical 30:481-484, nov-dez, 1997.

Faculdade de Medicina da Universidade de São Paulo.

Recorremos a duas preparações comerciais distintas, com mesmo princípio ativo e a seguir especificadas: A) "Ivermectin, MSD Ivomec": solução oral contendo $0,08 \% \mathrm{p} / \mathrm{v}$; uso veterinário; número da partida: 003/94; "Merck Sharp \& Dohme Farmacêutica e Veterinária Ltda." Souzas, Campinas, SP; B) "Ivermectine, MSD Mectizan"; comprimidos com 6mg; uso humano; lote: 881520W AVR96; "Laboratoires Merck Sharp \& Dohme", Paris, França.

Os animais infectados receberam inóculo de 2.000 larvas filarióides, por via subcutânea.

A dose de A e de B foi de $0,2 \mathrm{mg} / \mathrm{kg}$. Colocamos a preparação A em água destilada; quanto ao produto $\mathrm{B}$, após triturado, também, adicionamos água destilada. Constituímos sempre volume final de $0,5 \mathrm{ml}$ administrado uma única vez, oralmente, através de sonda esofágica.

Os roedores foram distribuídos em sete grupos: I- 5 animais infectados e tratados, após 24 horas, com A (solução); II- 5 animais infectados e tratados, após 24 horas, com B (comprimido); III- 5 animais infectados e tratados, após 8 dias, com A (solução); IV- 5 animais infectados e tratados, após 8 dias, com $\mathrm{B}$ (comprimido); V- 5 animais infectados e não tratados (controle); VI- 6 animais não infectados que receberam solução A, sendo que 3 deles, após 24 horas e os 3 restantes, depois de 8 dias; VII- 6 animais não infectados e que receberam a preparação B (comprimido), sendo que 3 deles, após 24 horas e os 3 restantes, depois de 8 dias.
A avaliação da eficácia ocorreu 12 dias após a infecção, por pesquisa das fêmeas partenogenéticas na parede intestinal dos animais dos Grupos I, II, III e IV, infectados e tratados, sucedendo comparação com o verificado nos do Grupo VI, controles de infectados e não tratados. Para quantificação dos helmintos recolhidos, sacrificamos os roedores e retiramos $15 \mathrm{~cm}$ do primeiro segmento do intestino delgado, que foi invertido, exteriorizando-se assim a mucosa intestinal. Imergimos estes fragmentos, durante 60 minutos, em tubos de ensaio contendo água aquecida a $42^{\circ} \mathrm{C}$. Em seguida, centrifugamos o sedimento obtido, a $2.500 \mathrm{rpm}$, por 2 minutos, e examinamos em microscópio entomológico (45X).

As taxas de eficácia das duas preparações derivaram de fórmula na qual RVA expressa a recuperação dos vermes adultos, tanto em Grupos infectados e tratados depois de 24 horas, fase de migração larvária, como naqueles infectados e tratados após 8 dias, em correspondência à fase adulta; assim:

eficácia $=\frac{\text { RVA }(\text { experimental })-\text { RVA }(\text { controle })}{\text { RVA }(\text { controle })}$

\section{RESULTADOS}

Os resultados obtidos e correspondentes às preparações A e B, dadas em dose única de $0,2 \mathrm{mg} / \mathrm{kg}$ por via oral, estão nas Tabelas 1,2 e 3, nas quais são perceptíveis significantes reduções do parasitismo.

Tabela 1 - Tratamento, por meio da ivermectina administrada por via oral, da estrongiloidiase experimental, em ratos parasitados pelo Strongyloides venezuelensis: informes sobre a infecçâo e a avaliaçâo.

\begin{tabular}{lccccc}
\hline Grupo & Fármaco & $\begin{array}{c}\text { Dose (única) } \\
\mathrm{mg} / \mathrm{kg}\end{array}$ & $\begin{array}{c}\text { Número de larvas usadas } \\
\text { para infectar }\end{array}$ & $\begin{array}{c}\text { Época de administração do } \\
\text { fármaco, após a infecção (dias) }\end{array}$ & $\begin{array}{c}\text { Época de sacrifĩcio dos animais, } \\
\text { após a infecção (dias) }\end{array}$ \\
\hline I & $\mathrm{A}$ & 0,2 & 2.000 & 1 & 12 \\
II & $\mathrm{B}$ & 0,2 & 2.000 & 8 & 12 \\
III & $\mathrm{A}$ & 0,2 & 2.000 & 8 & 12 \\
IV & $\mathrm{B}$ & 0,2 & 2.000 & & 12 \\
$\mathrm{~V}$ & Controle & & 2.000 & & 12 \\
\hline A = "Ivomec" (uso veterinário); B = "Mectizan" (uso humano).
\end{tabular}

Tabela 2 - Tratamento, por meio da ivermectina (A) administrada por via oral, da estrongiloidiase experimental, em ratos parasitados pelo Strongyloides venezuelensis: números de vermes recuperados dos intestinos.

\begin{tabular}{|c|c|c|c|c|}
\hline \multirow{2}{*}{$\begin{array}{c}\text { Época de administração do } \\
\text { fármaco, após a infecção } \\
\text { (dia) }\end{array}$} & \multicolumn{2}{|c|}{ Ivermectina $(0,2 \mathrm{mg} / \mathrm{kg})$} & \multicolumn{2}{|c|}{ Controle } \\
\hline & $\begin{array}{c}\text { Número total de vermes } \\
\text { adultos, em } 5 \text { ratos }\end{array}$ & $\begin{array}{c}\text { Número médio de vermes } \\
\text { adultos, por animal }\end{array}$ & $\begin{array}{l}\text { Número total de vermes } \\
\text { adultos, em } 4 \text { ratos* }\end{array}$ & $\begin{array}{c}\text { Número médio de vermes } \\
\text { adultos, por animal }\end{array}$ \\
\hline 1 & 310 & 62,0 & 1.575 & 393,7 \\
\hline 8 & 39 & 7,8 & 1.575 & 393,7 \\
\hline
\end{tabular}

$\mathrm{A}=$ "Ivomec" (uso veterinário).

* um animal morreu em virtude de causa indeterminada. 
Amato Neto V, Carignani FL, Matsubara L, Braz LMA. Tratamento de ratos, experimentalmente infectados pelo Strongyloides venezuelensis, através da ivermectina administrada por via oral. Revista da Sociedade Brasileira de Medicina Tropical 30:481-484, nov-dez, 1997.

Tabela 3 - Tratamento, por meio da ivermectina (B) administrada por via oral, da estrongiloidiase experimental, em ratos parasitados pelo Strongyloides venezuelensis: números de vermes recuperados dos intestinos.

\begin{tabular}{|c|c|c|c|c|}
\hline \multirow{2}{*}{$\begin{array}{l}\text { Época de administração do } \\
\text { fármaco, após a infecção }\end{array}$} & \multicolumn{2}{|c|}{ Ivermectina $(0,2 \mathrm{mg} / \mathrm{kg})$} & \multicolumn{2}{|c|}{ Controle } \\
\hline & $\begin{array}{l}\text { Número total de vermes } \\
\text { adultos, em } 5 \text { ratos }\end{array}$ & $\begin{array}{c}\text { Número médio de vermes } \\
\text { adultos, por animal }\end{array}$ & $\begin{array}{l}\text { Número total de vermes } \\
\text { adultos, em } 4 \text { ratos* }\end{array}$ & $\begin{array}{l}\text { Número médio de vermes } \\
\text { adultos, por animal }\end{array}$ \\
\hline 1 & 531 & 106,2 & 1.575 & 393,7 \\
\hline 8 & 800 & 160,0 & 1.575 & 393,7 \\
\hline
\end{tabular}

B = "Mectizan" (uso humano).

* um animal morreu em virtude de causa indeterminada.

Observou-se sensível superioridade $(84,2 \%)$ de A em relação à $\mathrm{B}(73,0 \%)$, quando consideradas as formas larvárias, e bem maior eficácia da primeira $(98,0 \%)$ a propósito dos vermes adultos (Tabela 4).

Tabela 4 - Tratamento, por meio de duas preparações de ivermectina administradas por via oral, da estrongiloidias experimental, em ratos parasitados pelo Strongyloide venezuelensis: comparação da eficácia quanto à eliminação de vermes adultos e de formas larvárias. Época de administração

\begin{tabular}{ccc} 
do fármaco, após a & Ivermectina (A)* & Ivermectina (B)* \\
infecção (dia) & $\%$ & $\%$ \\
\hline 1 & 84,2 & 73,0 \\
8 & 98,0 & 59,3
\end{tabular}

A = "Ivomec" (uso veterinário); B = "Mectizan" (uso humano).

* dose de $0,2 \mathrm{mg} / \mathrm{kg}$.

\section{DISCUSSÃO}

O sistema escolhido para averiguar a ação de medicamentos na estrongiloidíase foi a infecção experimental por $S$. venezuelensis em ratos, que permite ilações quanto ao tratamento da doença humana e de outros animais.

A utilização da ivermectina, mesmo em dose única, demonstrou significativa eficiência no tratamento da estrongiloidíase e, além disso, a tolerabilidade, em estudo não comparativo, sugeriu que ela seja menos tóxica do que o tiabendazol7. A potência de uma simples posologia contra diversos nematóides provou serem desnecessários regimes de tratamento com quantidades repetidas; ainda mais, em experimento não controlado o tratamento de helmintíases gastrintestinais com o composto citado, dado através de dose singela de $200 \mu \mathrm{g} / \mathrm{kg}$, revelou efetividade a respeito da infecção por Strongyloides e superioridade no confronto com albendazol ou mebendazol6.

Campos e cols 5 , em modelo experimental idêntico ao que empregamos, conseguiram eliminação total dos vermes, tratando com $0,5 \mathrm{mg} / \mathrm{kg}$ da ivermectina injetável 48 horas depois da inoculação de larvas.
$\mathrm{Na}$ investigação que empreendemos os animais infectados receberam o fármaco após 24 horas e 8 dias, para atingir no primeiro tempo as formas larvárias e, no segundo, as adultas, de acordo com o ciclo de vida do parasita. Pudemos constatar que A e B atuaram segundo diferentes intensidades na infecção de ratos pelo $S$. venezuelensis. O medicamento A foi superior quando avaliadas as atividades sobre vermes adultos ou larvas.

Impõe-se esclarecer uma diferença entre A e B, que são preparações que contêm o mesmo princípio ativo. O medicamento A corresponde a produto apresentado sob forma líquida, pronto para uso; o B, em comprimidos, requereu trituração e colocação em veículo (água destilada). Talvez essas circunstâncias tenham influído na absorção.

Como consideração final, julgamos lícito concluir que a ivermectina é anti-helmíntico bastante valioso se cogitado para o tratamento da estrongiloidíase. O procedimento que adotamos comprova isso, em apreciação que teve a intenção de analisar a utilidade de remédios administráveis por via oral, logicamente preferíveis operacionalmente. Utilizamos os dois produtos aqui disponíveis, incluindo o empregado em Medicina Veterinária. Comprovamos a eficácia, promissora quando desejada a cura da verminose por meio de conduta bem simples e, também, auspiciosa em virtude do efeito frente as larvas, muito almejado quando a parasitose é do tipo generalizado, disseminado e ainda rotulado como de hiperinfecção, hoje mais detectado em imunodeprimidos.

O produto para uso humano atualmente é fornecido, pela empresa produtora, apenas para tratamento da infecção oncocercótica, mas esse detalhe é meramente circunstancial e não impede que se cogite de prescrição quando presente a estrongiloidíase, sobretudo em sua modalidade grave. 
Amato Neto V, Carignani FL, Matsubara L, Braz LMA. Tratamento de ratos, experimentalmente infectados pelo Strongyloides venezuelensis, através da ivermectina administrada por via oral. Revista da Sociedade Brasileira de Medicina Tropical 30:481-484, nov-dez, 1997.

\section{SUMMARY}

Strongyloides venezuelensis experimental infection in rats was treated by two different oral preparations of ivermectin, $0.2 \mathrm{mg} / \mathrm{kg}$. One was a buman formula used by WHO in the treatment of onchocerciasis; the other was a veterinary preparation. Adult worms and larvae were evaluated. The human formulation cleared both forms in 59.3\% (adult worms) and
$73.0 \%$ (larvae), whereas the veterinary one cleared 98.0\% and $84.2 \%$, respectively. The antilarval action is very useful when treating systemic strongyloidiasis

Key-words: Strongyloides venezuelensis Experimental infection in rats. Ivermectin. Oral treatment.

\section{REFERÊNCIAS BIBLIOGRÁFICAS}

1. Aziz MA. Ivermectin vs onchocerciasis. Parasitology Today 2:233-235, 1986.

2. Brener Z, Chaia G. Isolamento e manutenção do Strongyloides ratti (Sandground, 1925) em condições de laboratório. Revista Brasileira de Biologia 20:447-451, 1960.

3. Campbell WC. Ivermectin, an antiparasitic agent. Medicinal Research Reviews 13:61-79, 1993.

4. Campbell WC, Fisher MH, Stapley EO, AlbersSchönberg G, Jacob TA. Ivermectin: A potent new antiparasitic agent. Science 221:823-828, 1983.

5. Campos R, Pinto PLS, Amato Neto V, Matsubara L, Miyamoto A, Carvalho SA, Takiguti CK, Moreira, AAB. Tratamento da infecção experimental de ratos por Strongyloides venezuelensis, mediante uso da ivermectina e do levamisol injetáveis. Revista do Instituto de Medicina Tropical de São Paulo 31:48-52, 1989

6. Freedman DO, Zierdt WS, Lujan A, Nutman TB.The efficacy of ivermectin in the chemotherapy of gastrointestinal helminthiasis in humans. The Journal of Infectious Diseases 159:1.151-1.153, 1989.

7. Naquira C, Jimenez G, Guerra JG, Bernal R, Nalin DR, Neu D, Aziz M. Ivermectin for human strongyloidiasis and other intestinal helminths. The American Journal of Tropical Medicine and Hygiene 40:304-309, 1989. 